\title{
GOÄ-Novelle
}

\section{Mengensteigerung im Privatlabor im Visier}

\author{
Privatärztlich zu erbringende Laborleistungen haben für Praxen \\ einen unbestreitbaren Reiz - zu viel Reiz meinen Kritiker und wollen \\ gegensteuern. Die GOÄ-Novelle könnte das Vehikel dafür sein.
}

aborleistungen für Privatversicherte sind derzeit - noch - einträglich. Die bevorstehende GOÄ-Novelle könnte das ändern. Manche Laborgemeinschaften leisten dem nach Meinung von Kritikern Vorschub. Der Stein des Anstoßes sind „Kostenlisten“, auf denen Laborgemeinschaften Kosten und Erlöse für Privatlaborleistungen aufführen. Damit sollen offenbar Mitglieder von anderen Laborgemeinschaften abgeworben werden. Die Erlöse pro Parameter erreichen fast durchweg zweistellige Beträge bis zu 20 EUR. Der Haken: Es handelt sich um Parameter des Speziallabors (GOÄ-Abschnitt M III und M IV).

\section{Beziehungsverbot gilt auch bei räumlicher Nähe}

Ärzte können aus Laborgemeinschaften die Leistungen des Basislabors, die in Abschnitt M II des Gebührenverzeichnisses gelistet sind, beziehen und als eigene Leistung abrechnen. Leistungen aus den Abschnitten M III und M IV jedoch müssen persönlich oder unter der ständigen Aufsicht des Arztes, der sie abrechnet, erbracht werden. Sie dürfen nicht aus Laborgemeinschaften bezogen und eigenständig berechnet werden. Das gilt, wie der Verband der Privaten Krankenversicherung (PKV) auf Anfrage mitteilt, nach seiner Auffassung auch dann, wenn ein Arzt seinen Sitz in räumlicher Nähe zur Laborgemeinschaft hat und sich damit zumindest theoretisch persönlich in die Laborgemeinschaft begeben könnte, um die Bedingungen für die Abrechenbarkeit von M-III- oder M-IV-Parametern zu erfüllen.
Dass in Preislisten auf die "entsprechenden Regularien der GOÄ" hingewiesen wird, ist aus Sicht von Kritikern (die sich dazu nur anonym äußern wollen) lediglich der Versuch, diese „kriminelle" Praxis zu bemänteln. Die große Mehrheit der Ärzte rechne Privatlaborleistungen korrekt ab, sagt ein Laborfachmann, doch durch einige wenige werde das System „für alle Ärzte ruiniert".

In der PKV sollen die Pro-Kopf-Ausgaben für ambulante Laborleistungen inzwischen das Viereinhalbfache der ProKopf-Ausgaben für gesetzlich Versicherte erreichen. In einem Diskussionspapier von 2009 bezeichnete das Wissenschaftliche Institut der PKV dies als unverhältnismäßig. Es sei nicht zu rechtfertigen, dass Niedergelassene einen erheblichen Teil ihres Mehrumsatzes bei Privatpatienten „durch extensive Veranlassung von Laborleistungen erzielen können“. Es gilt als sicher, dass die für 2011 anstehende GOÄ-Novelle auch der Mengensteigerung im Privatlabor entgegensteuern wird.

\section{Sind Preislisten Betrug?}

$\mathrm{Ob}$ der mit den Preislisten suggerierte vorschriftswidrige Bezug von Speziallabor-Parametern aus der Laborgemeinschaft Betrug darstellt, ist indessen rechtlich nicht geklärt. Für die Privatpatienten und ihre Krankenversicherer entsteht kein Vermögensschaden: Der Rechnungsbetrag ist derselbe, ob die Leistungen vom behandelnden, aber nicht zum Speziallabor berechtigten Arzt abgerechnet werden oder von einem Arzt, der die GOÄ-Vorschriften hierfür erfüllt. $\quad m p$ 